\title{
Aportes de la investigación social en salud: la importancia de la perspectiva de género en \\ la comprensión de la enfermedad coronaria
}

\section{Lucero Aída Juárez Herrera y Cairo ${ }^{1}$ María del Carmen Castro Vásquez²}

\section{Resumen}

El objetivo de este trabajo es discutir algunas aportaciones de la investigación social en salud desde la perspectiva de género al estudio de la enfermedad coronaria (EC), específicamente para el caso de las mujeres. Se asume que los modelos de interpretación desde esta perspectiva, posibilitan el estudio integral del proceso salud-enfermedad-atención (s/ e/a) de la EC, a la par que generan conocimiento sobre las desigualdades de género en salud; visibilizando los riesgos más allá de las diferencias biológicas; para identificar las maneras en que se auto-perciben y cuáles son las vivencias durante el proceso s/e/a de la EC, así como la toma de decisiones en salud. Estos estudios contribuyen a la búsqueda de estrategias de prevención y promoción para la EC.

Palabras claves: investigación social, perspectiva de género y enfermedad coronaria de mujeres. 


\begin{abstract}
This paper discusses the contributions made by social sciences, particularly from gender and socio cultural studies regarding coronary diseases (CD), specifically for women. It is assumed that the interpretation models for these perspectives enable the integral study of the health-diseasecare process for $\mathrm{CD}$, they also generate knowledge about gender inequality in health; visualizing risks beyond biological differences; to identify their self-perception and which are the experiences along the $\mathrm{CD}$ process, as well as decision taking regarding health. The study contributes to the search for preventive and promotion strategies for $\mathrm{CD}$.
\end{abstract}

Keywords: social investigation, gender perspective and coronary disease of women.

RECEPCIÓN: 23 DE MARZO DE 2013/ACEPTACIÓN: 31 DE AGOSTO DE 2015

\title{
La investigación social en salud desde la perspectiva de género: mujeres con enfermedad coronaria
}

La perspectiva de género abre el análisis a dimensiones que son frecuentemente soslayadas en los análisis biomédicos sobre la Enfermedad Coronaria (EC). Dicha mirada reclama la articulación de las desigualdades de género con otras variables como raza/etnia, clase social, edad y condiciones sociales específicas que colocan 
en mayor o menor vulnerabilidad a los diferentes grupos poblacionales, en particular al de mujeres.

A través de modelos interpretativos de las ciencias sociales y particularmente de los estudios de género, se enriquece la investigación, considerando que esta perspectiva de análisis, en tanto cuestionadora del estatus quo, visibiliza desigualdades sociales y sesgos de género en salud, que inician en la investigación y se reproducen en la práctica sanitaria (Ruiz-Cantero y Verdú-Delgado, 2004, Ruiz-Cantero et al., 2007).

El objetivo del trabajo ${ }^{3}$ es discutir algunas aportaciones de investigación social en salud al estudio de la EC de las mujeres, basándonos en trabajos publicados principalmente en México y América Latina.

Las enfermedades no son sólo entidades biológicas, sino que son también construcciones sociales, culturales e históricas que forman parte de un proceso salud/enfermedad/atención (proceso s/e/ a), social e históricamente situado, mediado por las estructuras sociales, pero vivido en las dinámicas individuales y colectivas (Laurell, 1982; Menéndez, 1994).

Los estudios de género y salud han demostrado que cuando la información sobre salud, enfermedad y atención médica se desagrega por sexo, se observan diferencias más o menos significativas, y frecuentemente se carece de explicaciones sobre la mayoría de esas diferencias, generando “...incertidumbre sobre la calidad de la información, sobre su validez para la planificación y sobre la efectividad y equidad de las actuaciones sanitarias" (Colomer-Revuelta, 
2007: 92). En México, la investigación social sobre EC con esta perspectiva, es aún muy limitada, a pesar de que en este problema subyacen diferencias de género en la población, sobre la construcción y percepción de riesgo coronario, y en las maneras de enfermar, atenderse y morir por EC (Tajer, 2009), así como entre el personal de salud al ofrecer la atención.

En el país, como en otras regiones del mundo, la EC tiene un peso creciente en la carga de la enfermedad y en la mortalidad general. Los primeros reportes de este comportamiento surgieron en la última década del siglo XX (Lozano-Ascencio et al., 1990; Frenk et al., 1991), tendencia que ha permanecido. Así, la EC se ubica como la primera causa de muerte en México, en hombres y mujeres (Escobedo et al., 2010; Lozano-Ascencio, 2013; INEGI, 2013).

En los 90`s, Ayanian y Epstein (1991) en los EEUU, reportaron diferencias en el manejo médico hospitalario de hombres y mujeres con EC, y señalaron que la enfermedad era más letal en ellas. En tanto que Healy (1991) evidenció que cuando las mujeres con EC llegaban a los servicios de salud, tenían que demostrar que podían padecer este tipo de enfermedades, y comportarse como hombres, para ser tratadas (como hombres) por el personal de salud, a lo que denominó síndrome de Yentl.

A raíz de estos reportes, se realizaron investigaciones en otros países confirmándose, que el personal de salud desarrollaba menos esfuerzos diagnósticos y terapéutico en mujeres que en hombres con EC (Rohlfs et al., 2004; Ruiz-Cantero y Verdú-Delgado, 2004; Tajer, 2002; Tajer et al., 2003), que había un notorio sub-registro de 
los primeros síntomas coronarios de las mujeres por parte del personal médico (Tajer, 2005), y que esos síntomas eran tratados frecuentemente con ansiolíticos, al atribuirlos a cuadros de ansiedad.

Así mismo se ha reportado que las manifestaciones de la EC de mujeres, suelen ser "inespecíficas" o "atípicas" (Solorio et al., 2006; Medrano, 2012; Almanza-Vega et al., 2014), lo que pudiera tener de fondo un sesgo en la investigación clínica al transpolar los resultados y estudios de varones (cuadro clínico "típico") a las mujeres. Esto contribuyó a que la enfermedad se construyera con base en el modelo masculino (Emslie et al., 2001; Tajer, 2005; Valls-Lobet, 2009) y la EC sea expresión de la mirada androcéntrica en la investigación científica biomédica (Ruiz-Cantero y Verbrugge, 1997).

La producción bibliográfica sobre EC de mujeres con un abordaje biomédico, es muy amplia. En la mayoría se asume que el pronóstico de las mujeres con EC es más sombrío, porque la padecen más tardíamente que los hombres, está relacionada con diferencias anatómico-fisiológicas, con los cambios metabólicos y hormonales de la menopausia, y porque las mujeres demoran la búsqueda de atención (Zárate et al., 2007, Solorio et al., 2006).

Desde la biomedicina se establece que a diferencia de los hombres, en las mujeres las arterias coronarias son más angostas, tienden a presentar menos lesiones obstructivas, son más frecuentes las alteraciones de la microcirculación y/o de la movilidad del músculo cardíaco, los procedimientos quirúrgicos son menos exitosos, y tienen más comorbilidad —con diabetes, obesidad e hipertensión 
arterial principalmente- (Pratt, 2007; Arrebola-Moreno et al., 2011; Cortes-Bergoderi et al., 2012).

Sin embargo, estas explicaciones no son suficientes para comprender las múltiples maneras en que se relacionan género y EC. Desde las ciencias sociales, como la sociología, antropología, psicología y desde las ciencias de la salud, como salud pública y enfermería, se ha reportado que si bien hay problemas comunes para hombres y mujeres con EC, también hay especificidades y particularidades propias de cada persona y/o colectivo, que imprimen diferencias como: a) los riesgos en salud y el modo (s) en que se construye la percepción de riesgo y la vulnerabilidad coronaria (Tajer, 2005, 2009, 2011, 2013); b) las creencias acerca del corazón y sus problemas (Emslie et al., 2001); c) la (s) manera (s) en que el imaginario social conforma barreras de género para la prevención de la EC y condiciona prácticas diferenciadas de atención (Tajer et al., 2014); d) las experiencias, afrontamiento y gestión ante la EC (Soto, 2007; Rincón, 2013; Mussi et al., 2013); e) la reproducción de sesgos de género en la atención de la EC (Ruiz-Cantero y Verdú-Delgado, 2004).

El género es una dimensión central en la vida humana y un organizador de la sociedad; en salud la construcción genérica expresa modos diferenciales de enfermar y morir. Sobre la EC se busca identificar cómo las mujeres acceden a la información, construyen su percepción de riesgo, viven la enfermedad y gestionan sus cuidados, porque a pesar de los avances en la atención de la EC, persisten desigualdades sociales que las impactan con mayor frecuencia (Velasco, 2010). 
Sobre la EC de mujeres se ha señalado que hay diversos grados de desconocimiento de ellas acerca del riesgo de padecer o de morir por EC y que es reducido el número de mujeres que siguen la directriz de cambiar el estilo de vida, como se recomienda desde la biomedicina (Manzur et al., 2005; TNS Gallup, 2010). Además, que aún cuando hay más mujeres con información acerca de factores de riesgo cardiovascular, síntomas coronarios y estrategias preventivas, ellas continúan identificando el cáncer de mama como el principal problema de salud de las mujeres, sobre todo las más jóvenes (Mosca et al., 2010; Rolandi et al., 2013).

La mayor visualización de los cánceres relacionados con la reproducción, pudiera explicarse por la actividad de los colectivos de mujeres que pusieron en la mesa de las discusiones la salud sexual y reproductiva, y la implementación de campañas preventivas desde las instituciones de salud. Pero estos reportes evidencian que hace falta visibilizar más la EC de mujeres, así como estrategias de información hacia las jóvenes. Si bien las mujeres acuden con mayor frecuencia a los servicios de salud por razones reproductivas, cuando inician con EC, su atención suele demorarse (Soto, 2007; Medrano, 2012).

\section{Imaginario y subjetividad}

Sobre esta temática, Emslie et al., (2001) señalan que dentro del imaginario social hay creencias sobre el corazón de las mujeres, de las que no está exento el personal de salud, como que "tienen más 
corazón", "padecen" más problemas sentimentales y emocionales y su corazón es más "blando" y "suave”, más romántico y amoroso. Sin embargo, desde la biomedicina se considera que estas son concepciones "no científicas" de la enfermedad. El problema es que por un lado se reproduce la naturalización social de las mujeres como "emocionales" (Valls-Lobet, 2006; Herrera, 2010), por el otro se puede dejar de lado la comprensión de factores psicosociales de riesgo cardiovascular, y también se deslegitiman saberes populares, individuales y colectivos, siendo dimensiones socioculturales y de género que intervienen en la comprensión, vivencia y atención de la EC.

Autores como Tajer (et al.) han estudiado en Argentina desde 1998 la construcción de identidad masculina y femenina y los modos de construcción de riesgo en salud; sobre el sub-registro de los síntomas coronarios por parte de las mujeres y de personal de salud; la identificación y comprensión de la vulnerabilidad coronaria diferencial, por género, edad y clase social; y sobre equidad de género y calidad de la atención en EC. Exploran también cómo se construyó en el imaginario social a la EC como una "enfermedad del género masculino" y cómo persiste esta construcción, a pesar del incremento de la enfermedad en la mujer (1998, 2000, 2002, 2003, 2005, 2010, 2011, 2013).

Tajer (2009) reporta que se invisibilizó el modo femenino de construcción de riesgo coronario, porque se generalizó el masculino como el válido y universal, con base en dos vertientes de producción-legitimación, el fenómeno de la "oveja negra" referido al 
mayor costo que presupone al sistema de salud el que una persona se enferme de una patología que no es frecuente (ni esperada) para su grupo social de pertenencia; y el "síndrome de Yentl" (Healy, 1991).

Entre las conclusiones más recientes de esta autora y colaboradoras (2014), señalan que hay avances en la percepción de riesgo psicosocial por parte de las mujeres, pero que hay distancias entre imaginarios y prácticas en salud, por parte de usuarias de servicios médicos y del personal de salud, con diferencias entre centros públicos y privados. Enfatizan la importancia de médicos/as generalistas y gineco-obstetras en la prevención primaria de la EC.

El trabajo de Almanza-Vega et al., aporta al campo de estudio de la EC en mujeres, al concluir sobre sus experiencias subjetivas en el manejo del dolor torácico explicando la experiencia del síntoma, estrategias de manejo del síntoma, y resultados del síntoma, desde los componentes fisiológicos, sicológicos y comportamentales; en este mismo tema, Céspedes $(2012,2013)$, también reportó resultados sobre percepción, evaluación y respuesta al dolor de mujeres con EC, pero al igual que Almanza et al., tampoco hace el análisis desde la perspectiva de género, aunque consideramos que aportan a identificar dichas diferencias entre hombres y mujeres.

Otros trabajos que explícitamente asumen que sus análisis se hacen desde la perspectiva de género, se han limitado solamente a señalar las diferencias entre hombres y mujeres. Entre éstos podemos mencionar al de Urquijo et al., (2003), que analizan características psicológicas de hombres y mujeres hospitalizados en terapia 
intensiva, con la finalidad de planear estrategias para llevar a cabo cambios conductuales sobre riesgos cardiovasculares. Entre sus conclusiones señalan que $92.8 \%$ de los y las pacientes eran "disfuncionales" por tener "trastornos de la personalidad". Pero, aquí debemos destacar un posible sesgo de género al afirmar que había una "fuerte" presencia de mujeres con "trastornos compulsivos".

En relación al estrés como factor de riesgo importante en la génesis de la EC, diversos estudios lo han señalado bajo la categoría de factores psicosociales, y otros íntimamente relacionados, como la depresión, ira y ansiedad (Dennolett y Brutsaert 1997-1998, citados por Tajer, 2009; Terrasa, 2011; Smith y Ruiz, 2002). Tajer et al., (2014) especifican factores que son estresores específicos del género femenino, relacionados con mandatos e ideales de género, altamente exigentes.

Otro estresor de género femenino es el estrés marital severo, pues de acuerdo a Orth-Gomér et al., (2000), empeora el pronóstico de mujeres afectadas por EC. Además, las mujeres suelen contar con menos apoyo de redes sociales que los hombres, en la fase de rehabilitación post infarto, lo que repercute en su sobrevivencia y calidad de vida (Orth-Gomér, 2009).

Consideramos que es necesario analizar desde la perspectiva de género los modelos que se utilizan en el estudio de los factores psicosociales de riesgo coronario, para que no invisibilice a las mujeres y se eviten sesgos al homogenizarse modelos construidos y validados con varones (Tajer, 2009). 


\section{Prácticas de cuidados}

En relación a las prácticas, las nociones estereotipadas sobre la EC permea la percepción de riesgo, la comprensión y significados de la enfermedad, y las maneras de gestionar y de afrontar este problema de salud, en tanto prácticas de cuidado que siguen una lógica de acuerdo a dinámicas y particularidades propias de cada individuo y colectividad.

Un problema de salud cuya experiencia es compleja, suele acompañarse del temor a morir, y compromete "ámbitos físicos, psicológicos y emocionales en la vida de la mujer" (Millán, 2009), detonando distintos procesos desde la negación a las estrategias de afrontamiento (Rincón, 2013). Esto son procesos de adaptación o de resistencia que influyen en las acciones en salud, como la búsqueda de atención médica, y el seguimiento de recomendaciones médicas sobre estilos de vida asociados a factores de riesgo cardiovascular, relativos a la alimentación, actividad física, tabaco, entre otros. Pero también devienen en sentimientos encontrados de falla, carga o debilidad, dadas las connotaciones del corazón en el imaginario social (Emslie et al., 2001), y las limitaciones que puede traer consigo la EC (Ledón, 2011).

En cuanto a la demora de las mujeres con EC para buscar atención médica, Mussi et al., (2013) analizaron diversos factores sociodemográficos y concluyeron que si bien las mujeres demoraron su llegada a servicios médicos en comparación con los hombres, la diferencia no era estadísticamente significativa. Los autores no 
abordaron dimensiones culturales ni ambientales de las diferencias.

Sobre las vivencias de las mujeres, Soto (2007) encontró que la demora para solicitar atención médica dependía de: la valoración que dan al dolor, la desprotección familiar de que suelen ser objeto, el que las mujeres privilegian el cuidado de otros sobre sus propias necesidades, su dependencia a terceros, la incapacidad para modificar su entorno, y que también las experiencias negativas con el personal de salud influyen en este aplazamiento. Aunque la autora no explicitó la utilización de la perspectiva de género en su análisis, su estudio tiene gran potencial descriptivo y los factores que identifica como explicaciones de la demora de las mujeres, se relacionan con patrones y estereotipos de género, inmersos en relaciones de poder que se reproducen en el cuidado de la salud.

Mientras Medrano (2012) señala, que si bien las mujeres priorizan la atención de otros antes que de sí mismas, la experiencia de vivir su cuerpo a través del dolor por la EC, las lleva a cuidarse, aunque sea temporalmente.

\section{Sesgos de género en la atención de la EC}

La investigación sociocultural y de género ha documentado también diversos sesgos en la atención a la salud cardiovascular por el género, la edad y la clase social, en base a la reconstrucción de las trayectorias de atención, y de reconocer que los factores que intervienen para que la letalidad sea más alta en mujeres, no son sólo 
de índole biológica (Ruiz-Cantero y Verbrugge, 1997; Ruiz-Cantero y Verdú-Delgado, 2004; Ruiz-Cantero et al., 2007), como se ha venido sosteniendo a lo largo de este trabajo. Los sesgos de género se producen por la

... creencia errónea de que los hombres y las mujeres son iguales en la manifestación de signos y síntomas precoces y tardíos de las enfermedades, iguales respondiendo a los tratamientos, y en sus pronósticos, cuando de hecho no se comportan de la misma forma, y que llevan a no detectar el problema de salud en uno de los sexos, en este caso (de la EC), en perjuicio de las mujeres (Ruiz-Cantero 2015: 6).

Sobre los sesgos de género en los esfuerzos diagnósticos y terapéuticos en la EC de mujeres (Ayanian y Epstein, 1991; Rohlfs et al., 2004), se ha reportado que hay avances en la comprensión de algunas dimensiones del problema (Tajer, et al., 2014), lo que no implica necesariamente que se reflejen en las prácticas de atención en los servicios de salud.

Uno de los sesgos que suele acompañar a los de género, es el generacional, al menos en dos sentidos, por un lado cuando se atribuye la letalidad diferencial de la enfermedad, sólo a la brecha entre hombres y mujeres en la edad de aparición de los síntomas — 6-10 años-, reportándose incluso que la prescripción de medicamentos utilizados para la prevención secundaria de la enfermedad, es menor para las mujeres debido a su "mayor edad", y esto podría expli- 
car parte de la mortalidad diferencial en la población femenina (Williams et al., 2003).

El otro refiere a mujeres menores de 50 años con EC, en quienes se complica aún más la identificación temprana de sus síntomas, tanto por ellas mismas cómo por el personal de salud. Como asienta la Fundación Favaloro, uno de los mitos sobre la EC de mujeres, es que no afecta a mujeres antes de la menopausia. Si bien es cierto que su incidencia es menor en mujeres jóvenes, también lo es que cuando la desarrollan, su probabilidad de morir sigue siendo más alta en comparación a los hombres de la misma edad.

El sesgo de género también se relaciona con la clase social, ya que la subestimación y el sub-registo de la EC, es más frecuente en mujeres de estrato socioeconómico bajo o medio bajo, cuyas premisas de género suelen ser más tradicionales (Tajer, 2009).

Otro sesgo tiene que ver con la idea de que las candidatas a padecer EC son mujeres "masculinizadas", en tanto están inmersas en la competitividad y complejidad del mundo moderno. Tajer (2009) observa que la vulnerabilidad coronaria de estas mujeres se asocia con los niveles de responsabilidad laboral, su poco poder para tomar decisiones en el espacio público, y con la doble jornada que llevan a cabo.

Uno más se relaciona con las decisiones diagnósticas en atención sanitaria, de acuerdo al género del personal médico. Adams et al., (2008), señalan que las médicas tendían a escuchar más ampliamente las historias acerca de la presentación de la enfermedad, particularmente cuando atendían a mujeres; los médicos 
aparentemente no mostraron diferencias entre hombres y mujeres en este aspecto.

Como hemos mostrado en párrafos anteriores, la investigación social aplica herramientas teóricas y metodológicas que complementadas con perspectiva de género, logran identificar diferencias respecto a los varones en la percepción del riesgo de las mujeres con EC, la vivencia de la misma y el impacto que tiene en la vida personal y familiar, partiendo de la premisa de género que enfatiza el papel central de la mujer como cuidadora de otros, antes que a sí misma.

\section{Algunas reflexiones para la discusión}

La perspectiva de género aporta una mayor y precisa problematización respecto a cómo las mujeres acceden a la información sobre la EC de mujeres, viven la enfermedad y gestionan sus cuidados, porque, hay que enfatizar, a pesar de los avances en la atención sanitaria, persisten desigualdades sociales en salud que las impactan con mayor frecuencia; se entiende que están más expuestas a riesgos y vulnerabilidades, relacionadas principalmente con la inequidad en el acceso a los servicios sanitarios o en la información en salud, por cuestiones sociales, culturales y económicas (OMS, 2009).

Las mujeres con EC viven a partir del diagnóstico, en la sujeción y obligación a nuevos modos de vida, al tener connotaciones profundas por las limitaciones físicas y psicológicas que conlleva el diagnóstico, como problemas en la realización de actividades de la 
vida cotidiana, en la reincorporación al trabajo, en la actividad sexual, en actividades sociales y de ocio. Lo que se conjuga con ansiedad, depresión, negación o sobredimensión de las limitaciones propias de la enfermedad, y elementos de dependencia y pasividad al adoptar el rol de enfermas (Peña, 2012).

En este punto cabe agregar, que los padecimientos cardiológicos afectan los roles de género de hombres y mujeres. En el caso de las mujeres, tienen implicaciones profundas relacionadas con el ya de por sí menor valor "productivo" de los roles femeninos tradicionales, al no poder cumplir con esos roles en tanto mujeres-enfermas, se refuerza el menor valor social de las mujeres, eso incluye a las que insertas en el mercado laboral, llevan a cabo la doble jornada, y/o tienen trabajos de "menor" importancia.

En cuanto a los cambios para mejorar la salud cardiovascular, el concepto "estilos de vida", al que se refiere la biomedicina, reduce la construcción colectiva y sociocultural de la mayoría de los factores de riesgo para la EC. La responsabilidad recae en el individuo y deja de lado la articulación entre las condiciones de vida, sociales, educativas, económicas, culturales, políticas y los patrones de conducta (Menéndez, 1998). Aunado a esto, hay una ausencia en el reconocimiento de la construcción de género entre hombres y mujeres ante la EC.

En el campo médico se articulan discursos históricos de feminidad, que enfatizan el "carácter corporal, sensible e irracional de las mujeres" (Herrera, 2010: 76), arquetipo que influye en la relación médico-paciente, lo que puede llevar al sub-registro de los 
primeros malestares de las mujeres con EC, minimizar la gravedad que puede estar presente en este tipo de patologías, así como aplazar el tratamiento y dejarlas fuera de ciertos procedimientos, reproduciéndose prácticas diferenciadas de atención (Tajer et al., 2014)

Utilizar la perspectiva de género, necesariamente implica un análisis relacional que lleva a identificar los elementos que perpetuán las desigualdades sociales en salud. Para Sen et al., (2005), la investigación social en salud enfrenta diversos retos, como evitar el sesgo de la biología, analizando la complejidad de las relaciones biológicas y sociales, así como identificando los condicionantes sociales que exacerban vulnerabilidades biológicas.

Centralizar el estudio de la EC de mujeres sólo en aspectos biológicos, como la relación con la menopausia, la genética, y la comorbilidad con factores de riesgo cardiovascular (obesidad, sedentarismo, hábitos alimentarios, hipercolesterolemia) y con enfermedades (hipertensión arterial y diabetes, principalmente) (Zárate et al., 2007; Cortes-Bergoderi et al., 2012), muestra limitaciones para incorporar otras dimensiones a las conductuales, y subestima las condiciones socioculturales, contextuales y de género de las personas y grupos sociales; es decir, los determinantes sociales de la salud, que difieren del enfoque tradicional de los factores de riesgo (Álvarez, 2009).

En relación al estrés, hay que cuestionar la etiquetación de las mujeres como "naturalmente" más emocionales, que puede llevar a tratar sus malestares con ansiolíticos y sub-registrar los primeros 
síntomas coronarios (Tajer, 2009). Habría que cuestionar qué es lo que se entiende por estrés desde los estudios biomédicos y reconocer los aportes desde otras disciplinas. Del mismo modo evidenciar que en el imaginario médico y colectivo, la EC permanece identificada como enfermedad masculina (Tajer, 2009; Tajer et al., 2014).

Es importante mencionar que los sesgos de género en la investigación pueden llevar a la exclusión sistemática de mujeres en los ensayos clínicos y que en el caso de la EC, esta exclusión puede articularse con el modo en que las mujeres construyen y perciben el riesgo coronario, sus experiencias, así como las estrategias y recursos con que cuentan o los obstáculos que enfrentan para acceder a los servicios de salud (Ruiz-Cantero et al., 2007; Valls-Lobet, 2009; Tajer et al., 2014).

Durante la revisión de trabajos para sustentar las temáticas presentadas, se descartaron los que si bien hacen referencia a diferencias de género, reducen la categoría a la variable "sexo", rescatando sólo algunos por su contribución puntual a los temas. En este sentido se observó que uno de los errores más comunes en trabajos biomédicos, es substituir la categoría o variable sexo por género y considerarlo un análisis de género, sin reconocer que son constructos diferentes. Esto se ha venido discutiendo tanto desde las ciencias sociales como de las de salud (Krieger, 2003; Salas-Valenzuela y Medina-Mora, 2006; Ruiz-Cantero et al., 2006; Gahagan et al., 2015).

Es importante reconocer que la dificultad para identificar la relación entre género y salud y entre EC y género, se vincula con la formación médica profesional, de ahí la impostergable necesi- 
dad de transversalizar género en las currículas universitarias, potencializando la enseñanza y la investigación en salud bajo la perspectiva de género.

En el caso de la EC en mujeres, se pugna por incorporar la transversalidad de género en las estrategias preventivas, los programas de salud y las políticas, que informen, empoderen y posibiliten las posibilidades de acción de las mujeres ante la EC, así como se trabaje con el personal de salud sobre género, derechos y calidad de la atención.

Queda pendiente, entre otros aspectos, identificar y analizar cómo la tardanza en reconocer los problemas de salud de las mujeres, se relaciona con una condición de ciudadanía que invisibiliza desigualdades, las subordina al discurso especializado del personal médico y limita sus procesos de autonomía. Y buscar innovaciones de género que aporten a la resolución de inequidades como se propone desde el grupo Gendered Innovations (Schiebinger, 2015).

Agradecimientos: CONACYT México por la Beca para estudios doctorales. A El Colegio de Sonora.

\section{Bibliografía}

AdAms, Ann; Buckingham, Chistopher; LindenMeYer, Antje; Mckinlay, Jonh; Link, Carol; MARCEAU, Lisa; ARbER, Sara (2008). "The influence of patient and doctor gender on diagnosing coronary heart disease", Sociology of Health Illnes, núm. 1, vol. 30, pp. 1-18. 
Almanza-Vega, Nanchy; Romero-Massa, Elizabeth; Espitia-MejíA, Mayuriz y Álvarez-Romero, Erich (2014). "Percepción, evaluación y respuesta al síntoma angina de pecho, por mujeres que presentaron síndrome coronario agudo", Revista Ciencias biomédicas, núm. 2, vol. 5, pp. 272-280.

Álvarez, Luz (2009). "Los determinantes sociales de la salud: Más allá de los factores de riesgo", Rev Geren Polit Salud, Bogotá, Colombia, núm. 17, vol. 8, junio-diciembre, pp. 69-79.

Ayanian, John y Epstein, Arnold (1991). "Differences in the use of procedures between women and men hospitalized for coronary heart diseases". New England Journal Medicine, vol. 325, pp. 221-225.

Arrebola-Moreno ${ }_{2}$ Antonio; Recio-Mayoral, Alejandro y Kaski, Juan (2011). "Papel de la microcirculación coronaria en las enfermedades cardiacas”. Cardiocore núm. 4, vol. 46, pp. 161-166.

CÉsPedes-CUEVAS, Viviana (2012). "Investigación que prueba teoría: una aproximación desde enfermería cardiovascular”. Aquichan, núm. 2, vol. 12, pp. 97-108.

— (2012). "Síntomas en la mujer con síndrome coronario agudo: desde las pruebas de validez". Avances en Enfermería, núm. 2, vol. 30, pp. 25-38.

Colomer-Revuelta, Concepción. (2007). "El sexo de los indicadores y el género de las desigualdades”. Revista Española de Salud Pública, núm. 2, vol. 81, pp. 91-93.

Cortes-Bergoderi, Mery; ThOmas, Randal; AlbuQuerque, Felipe; BAtsis, John; Burdiat, Gerard; Perez-Terzic, Carmen; Trejo-Gutiérrez, Jorge y LOPEZ-JIMENEZ, Francisco (2012). "Validity of cardiovascular risk 
prediction models in Latin America and among Hispanics in the United States of America: a systematic review". Revista Panamericana de Salud Pública, núm. 2, vol. 32, pp. 131-139.

Emslie, Carol; Hunt, Kate y Watt, Graham (2001). "Invisible women? The importance of gender in lay beliefs about heart problems", Sociology of Heath Illnes, núm. 2, vol. 23, pp. 203-233.

Escobedo, Jorge; RodRIGUEZ, Gabriela y BuITRÓN, Luisa (2010). "Morbilidad y mortalidad por Cardiopatía Isquémica en el Instituto Mexicano del Seguro Social. Estudio ecológico de tendencias en población amparada por el Instituto Mexicano del Seguro Social entre 1990 y 2008", Arch Cardiol Mex, núm. 4, vol. 80, pp. 242-248.

Frenk, Julio; Bobadilla, José; Stern, Claudio; Freika, Tomás y Lozano, Rafael (1991). "Elementos para una teoría de la transición en salud", Salud Pública de México núm. 5, vol. 33, septiembre-octubre, pp. 448462.

FundACIÓN FAVALORO. "Mitos y realidades acerca de la salud de la mujer" (s/f). En línea. Disponible en https://www.fundacionfavaloro.org/ educa_mitos_salud_mujer.html

GaHagan, Jacqueline; Gray, Kimberly y Whynacht, Ardath (2015). "Sex and gender matter in health research: addressing health inequities in health research reporting", International Journal for equity in health, núm. 1, vol. 14, pp. 1-4.

Healy, Bernardine (1991). "The Yentl syndrome". The New Journal of Medicine July, núm. 4, vol. 325, pp. 274-275.

Herrera, Cristina (2010). "De 'pacientita' a ciudadana: el largo sinuoso camino de las mujeres dentro del orden médico en México". En Poder 
médico y ciudadanía: el conflicto social de los profesionales de la salud con los derechos reproductivos en América Latina, Roberto Castro y Alejandra Gómez Editores. Montevideo: Comité Sectorial de Investigación Científica, pp. 75-99.

Instituto Nacional de Estadística y Geografía de México (INEgi) (2013). Mujeres y hombres en México 2013. En línea. Disponible en http://www.colpos.mx/wb/images/Meg/pdf/Myh_2013.pdf

Krieger, Nancy (1994). "Epidemiology and the web of causation: Has anyone seen the spider?". Soc Sci Med, núm. 7, vol. 39, pp. 887-903.

Laurell, Asa Cristina (1982). "La salud-enfermedad como proceso social", Revista latinoamericana de Salud, núm. 1, vol 2, pp. 7-25.

LEDÓN, Loraine (2011). "Articulación entre las categorías cuerpo, género y enfermedad crónica desde diferentes experiencias de salud", Revista cubana de salud pública, núm. 3, vol. 37, pp. 324-337.

Lozano-Ascencio, Rafael; Escamilla, José; Escobedo de la Peña, Jorge y López-Cervantes, Malaquia (1990). "Tendencia de la mortalidad por cardiopatía isquémica en México, de 1950 a 1985”, Salud Pública de México, núm. 4, vol. 32, pp. 405-415.

—; GÓMEZ-DANTÉs, Héctor; GARRIDO-LATORRE, Francisco; JimÉNEZ-COrona, Aída; CAmpuzANo-Rincón, Julio César; Franco-Marina, Francisco y Murray, Christopher (2013). "La carga de enfermedad, lesiones, factores de riesgo y desafíos para el sistema de salud en México”, Salud Pública de México, núm. 6, vol. 55, pp. 580-594.

Manzur, Fernando, Arrieta, Carlos et al., (2005). "Estudio sociológico y del conocimiento de los factores de riesgo de las enfermedades cardiovasculares en la Costa Caribe Colombiana" (Estudio 
Caribe). Revista Colombiana de Cardiología, núm. 3, vol. 12, pp. 122 128.

Medrano, Patricia (2012). Caracterización de los síntomas de Angina en mujeres hospitalizadas en la Clínica San Juan de Dios Cartagena. Tesis doctoral. Cartagena: Universidad Nacional de Colombia.

MenéNDEZ, Eduardo (1994). "La enfermedad y la curación? Qué es medicina tradicional" Alteridades, núm. 7, vol. 4, pp. 71-83.

. (1998). "Estilos de vida, riesgos y construcción social. Conceptos similares y significados diferentes”, Estudios Sociológicos, núm. 46, vol. 16, enero-abril, pp. 37-67.

Millán, Eillen y DíAz, Luz (2009). "Experiencia del dolor torácico en la mujer con infarto del miocardio", Avances en Enfermería, núm. 2, vol. 27, pp. 33-42.

Mosca, Lori; Mochari-Greenberger, Heidi; Dolor Rowena, Newby Kristin y RoB, Karen (2010). "Twelve-year follow-up of american women's awareness of cardiovascular disease risk and barriers to hearth health", Circulation cardiovascular Qual Outcomes núm. 2, vol. 32, march, pp. 120-127.

Mussi, Fernanda; Gibaut, Mariana; Damasceno, Carla; Mendes, Andreia; Guimaraes, Armenio y Santos, Carlos (2013). "Sociodemographic and clinical factors associated with the decision time for seeking care in acute myocardial infarction", Revista latino-americana de enfermagem, núm. 6, vol. 21, pp. 1248-1257.

Organización Mundial de la Salud [OMS] (2009). "Las Mujeres y la Salud. Los datos de hoy, La agenda del mañana”. En línea. Disponi- 
ble en http://whqlibdoc.who.int/hq/2009/WHO_IER_MHI_ STM.09.1_spa.pdf

Orth-Gomér, Kristina (2009). "Are social relationship less health protective in women that in men? Social relations, gender and cardiovascular health" Journal of Social $\mathcal{E}$ Personal Relationship, núm. 1, vol. 26, pp. 63-71.

- Wamala, Sara; Horsten, Myriam; Schenck-Gustaffson, Shneiderman Neil y Mittleman, Murray (2000). "Marital stress worsen prognosis in women with coronary heart disease" JAMA núm. 23, vol. 284, december, pp. 3008-3014.

PraTt, Guillermo (2007). Estudio anatómico de las arterias coronarias y su implicancia clínico-quirúrgica, Tesis Doctoral, Argentina: Facultad de Ciencias Médicas, Universidad Nacional de La Plata.

PeÑa, Carlos; Ramírez, José y Castro, Fernando (2012). "Infarto agudo del miocardio por estrés laboral”. Medicina Legal de Costa Rica, num. 2, vol. 29, pp. 111-119.

Rincón, Fanny (2013). La enfermedad coronaria en la mujer. Un asunto de cuidado, [ebook]. Universidad de Colombia.

RoHLFs, Izabella; García, María del Mar; Gavaldà, Laura; Medrano, María José; Juvinyà, Dolors; Baltasar, Alicia y Muñoz, Dolors (2004). Género y cardiopatía isquémica. En Gaceta Sanitaria, núm. 2, vol. 18, pp. 55-64.

Roland, Florencia; Grinfeld, Liliana; Sosa, María;Tambussi, Ana; Kazelian Lucía; La Bruna María et al., (2013). "Encuesta de reconocimiento de la enfermedad cardiovascular en la mujer por la mujer", Rev Argent de Cardiol núm. 4, vol. 81, pp. 330-335. 
Ruiz-Cantero, María Teresa y Verbrugge, Lois (1997). "A two way view of gender bias in medicine", Journal of Epidemiology and Community Health, núm. 2, vol. 51, pp. 106-109.

Ruiz Cantero, María Teresa y Verdú-Delgado, María (2004). "Sesgo de género en el esfuerzo terapéutico", Gac Sanit, núm. 1, vol. 18, pp. 118-125.

Ruiz-CANTero, María Teresa; SimÓN-Rodriguez, Elena y PaPí-Galvéz, Natalia (2006). "Sesgos de género en el lenguaje de los cuestionarios de la Encuesta Nacional de Salud 2003", Gacet Sanit núm. 2, vol. 20, pp. 161-165.

Ruiz-Cantero, María Teresa; Vives-Cases, Carmen; Artazcoz, Lucía; Delgado, Ana; García, María del Mar; Midueo, Consuelo; Montero, Isabel; OrTiz, Rocío; Ronda, Elena; Ruiz, Isabel y Valls, Carme (2007). "A framework to analyse gender bias in epidemiological research", J Epidemiol Community Health núm. 2, vol. 61, pp. 46-53.

Ruiz-Cantero, María Teresa (2015). Innovaciones de género para la salud pública: Universidad de Alicante. Lección inaugural curso académico 2015-2016. En línea. Disponible en http://web.ua.es/es/protocolo/documentos/eventos/apertura-curso/apertura-1516/ leccion-inaugural-apertura-curso-academico-2015-2016.pdf

Schiebinger, L.; Klinge, I.; SÁnchez de Madariaga, I.; Schraudner, M. and Stefanick, M. (Eds). (2011-2013). "Gendered innovations in Science, Health \& Medicine, Engineering, and Environment" (genderedinnovations.stanford.edu). En línea. Disponible en https:/ /genderedinnovations.stanford.edu/ 
Salas-Valenzuela, Monserrat y Medina-Mora, María del Pilar (2006). "El concepto género en documentos gubernamentales sobre salud y nutrición en México", Salud Colectiva, núm. 1, vol. 2, enero-abril, pp. 35-45.

Sen, Gita; Asha, George y Östlin, Piroska (2005). "Incorporar la perspectiva de género en la equidad en salud: un análisis de la investigación y la política”, Publicación ocasional, vol. 14, OPS.

Smith, Timothy y RuIz, John (2002). "Psychosocial influences on the development and course of coronary heart disease: Current status and implications for research and practice". Journal of Consulting and Clinical Psychology, núm. 3, vol. 70, pp. 548-568.

Solorio, Sergio; Hernández, Martha; RAngel, Alberto y Murillo-Ortiz, Blanca (2007). "Cardiopatía isquémica en mujeres mexicanas", Arch Cardiol Mex, núm. 3, vol. 77, pp. 226-231.

Soто, Alejandra (2007). "Factores que intervienen en la demora de la solicitud de atención médica o de enfermería en mujeres que presentan dolor toráxico de origen coronario", Av. enf. XXI, núm. 2, vol. 25, pp. 58-64.

Tajer, Debora; Cernada, J.; Barrera, Isabel y AlberTano, P. (1998). Mujeres, varones y enfermedad cardiovascular. Aportes epidemiológicos desde la salud mental y la perspectiva de género a la explicación de la incidencia diferencial por sexos de enfermedades cardiovasculares en adultos jóvenes. Universidad de Buenos Aires, Instituto de Investigaciones. Facultad de psicología. Programa UBCy T. Categoría jóvenes.

TAjer, Debora; Cernadas, J.; Barrera, Isabel ; Imaz, I. y Lo Russo, A. (2000). Género y construcción del riesgo en enfermedades cardiovasculares 
en adultos jóvenes. Universidad de Buenos Aires. Instituto de Investigaciones. Facultad de Psicología. Programa UBACyT. Categoría investigadores jóvenes.

Tajer, Debora; Fernández, Ana; Barreras, Isabel; Cernadas, M.I.; Lo Russo, Alejandra; GabA, M.; Idigoras, N. y Atardo, C. (2002). Equidad de género en la calidad de la atención en los pacientes cardiovasculares. Facultad de Psicología, x Anuario de Investigaciones, Universidad de Buenos Aires.

(2003). Equidad de género en la calidad de la atención en los pacientes cardiovasculares, 2a. Parte, XI Anuario de investigaciones. Facultad de Psicología, Universidad de Buenos Aires.

TAJER, Débora (2005). Mujeres y enfermedad cardiovascular. Género y subjetividad en la construcción del riesgo en enfermedades cardiovasculares en mujeres jóvenes. Síntesis de Tesis de Maestría. Universidad de Buenos Aires: Secretaría de Investigaciones/Anuario de investigaciones. Ciencias Sociales en Salud. Facultad de Psicología, pp. 239-248.

- (2009). Heridos corazones. Vulnerabilidad coronaria en hombres y mujeres. Buenos Aires, Paidós.

—- Cernadas, J.; BarRera, Isabel; Reid, G.; Lo Russo, A. y GabBa, M. y Attardo, C. (2010). "Equidad de género en la calidad de atención de pacientes cardiovasculares”. En Desigualdades sociales y equidad en salud: perspectivas internacionales, Guillermo González-Pérez, comp. Guadalajara: Universidad de Guadalajara.

TAjer, Debora; Gaba, Mariana y BARrera, Isabel (2011). Análisis de la situación de vulnerabilidad coronaria y género en la ciudad de Buenos Aires. 
Buenos Aires: Dirección General de la Mujer, Ministerio de Desarrollo Social del Gobierno de la Ciudad de Buenos Aires.

Tajer, Debora; ReID, Graciela; GABA, Mariana; Lo Russo, Alejandra y BARRERA, Isabel (2013). "Investigaciones sobre género y determinación psicosocial de la vulnerabilidad coronaria en varones y mujeres". Rev Argent Cardiol, núm. 4, vol. 81, pp. 344-352.

Tajer, Debora; Fernández, Ana; Antonietti, Laura; Chiodi, Agostina; SAlAZAR, Adriana; BARrerA, Isabel y JuÁREZ, Lucero (2014). "Barreras de género en la prevención cardiovascular. Actitudes y conocimiento de profesionales de la salud y de usuarias", Rev Arg Sal Pub, núm. 5, vol. 21, pp. 14-23.

TERRASA, Sergio (2011). "Actualización: factores psicosociales y su relación con la enfermedad cardiovascular". Evidencia. Actualización en la práctica ambulatoria, octubre-diciembre, pp. 149-152.

TNS GALLUP (2010). Estudio entre mujeres. Conocimiento sobre el riesgo en las enfermedades cardiovasculares. TNS Gallup Argentina.

Urquijo, Sebastian; Giles, Mariela; Richard’s, María y Pianzola, Enrique (2003). "Variables psicológicas asociadas a las patologías isquémico-vasculares. Un estudio de género”, Rev. Fed. Arg. Cardiol, núm. 32, pp. 190-198.

VALls-Lobet, Carmen (2006). Mujeres invisibles. Barcelona: Instituto de la Mujer: Random House Mondadori.

_ (2009.) Mujeres, salud y poder. Barcelona: Universitat de Valencia. Instituto de la Mujer: Ediciones Cátedra.

VELASCO, Sara (2010). Informe sobre metodología y determinantes psicosociales de género en enfermedades cardiovasculares. Para la revisión del plan de 
prevención de Enfermedades cardiovasculares de la comunidad Valenciana. Dirección General de Salud Pública (Consellería de Sanitat. Generalitat Valenciana).

Williams, David; Bennet, Kathleen y Feely, John (2003). "Evidence for an age and gender bias in the secondary prevention of ischemic heart disease in primary care", Br J Clin Pharmacol, núm. 6, vol. 55, pp. 604608.

ZÁrate, Arturo; SAUCEdo, Renata; BASURTo, Lourdes y Martínez, Carlos (2007). "La enfermedad cardiovascular como amenaza actual para la mujer adulta mayor. La relación con los estrógenos" Ginecología y Obstetricia de México núm. 5, vol. 75, 286-292. 